\title{
Les trois dimensions de la gouvernance coopérative agricole
} Le cas des coopératives vinicoles du Languedoc-Roussillon

The three dimensions of agricultural cooperative governance

The case of the wine-making cooperatives in

Languedoc-Roussillon

Las tres dimensiones de la gobernanza cooperativa agrícola

El ejemplo de las cooperativas vinícolas del

Languedoc-Rosellón

\section{Louis-Antoine Saïsset}

Numéro 339, janvier 2016

URI : https://id.erudit.org/iderudit/1035585ar

DOI : https://doi.org/10.7202/1035585ar

Aller au sommaire du numéro

\section{Éditeur(s)}

Association Recma

ISSN

1626-1682 (imprimé)

2261-2599 (numérique)

Découvrir la revue

Citer cet article

Saïsset, L.-A. (2016). Les trois dimensions de la gouvernance coopérative agricole : le cas des coopératives vinicoles du Languedoc-Roussillon. Revue internationale de l'économie sociale, (339), 19-36.

https://doi.org/10.7202/1035585ar
Résumé de l'article

Les coopératives agricoles, qui constituent un mode organisationnel original, subissent de profondes restructurations. Dans le secteur viticole en Languedoc-Roussillon notamment, les types de gouvernance sont en pleine évolution. L'objet de notre travail est d'analyser et de caractériser ce système de gouvernance spécifique, à travers l'étude qualitative de vingt-cinq entretiens avec des dirigeants de coopératives vinicoles du Languedoc-Roussillon. Les principaux résultats montrent l'originalité des organes de gouvernance des coopératives agricoles, se caractérisant par des relations complexes. En outre, grâce à l'analyse textuelle des retranscriptions d'entretiens, nous montrons que la gouvernance coopérative agricole comporte trois dimensions complémentaires dont l'importance est variable d'une coopérative à l'autre : dimension disciplinaire, dimension partenariale et dimension cognitive. 


\section{LES TROIS DIMENSIONS DE LA GOUVERNANCE COOPÉRATIVE AGRICOLE LE CAS DES COOPÉRATIVES VINICOLES DU LANGUEDOC-ROUSSILLON}

\section{Par Louis-Antoine Saïsset*}

*UMR MOISA 1110 Montpellier SupAgro.

Les coopératives agricoles, qui constituent un mode organisationnel original, subissent de profondes restructurations. Dans le secteur viticole en Languedoc-Roussillon notamment, les types de gouvernance sont en pleine évolution. L'objet de notre travail est d'analyser et de caractériser ce système de gouvernance spécifique, à travers l'étude qualitative de vingtcinq entretiens avec des dirigeants de coopératives vinicoles du Languedoc-Roussillon. Les principaux résultats montrent l'originalitédes organes de gouvernance des coopératives agricoles, se caractérisant par des relations complexes. En outre, grâce à l'analyse textuelle des retranscriptions d'entretiens, nous montrons que la gouvernance coopérative agricole comporte trois dimensions complémentaires dont l'importance est variable d'une coopérative à l'autre: dimension disciplinaire, dimension partenariale et dimension cognitive.

\section{The three dimensions of agricultural cooperative governance:}

\section{The case of the wine-making cooperatives in Languedoc-Roussillon}

Agricultural cooperatives, which are a unique organisational form, are undergoing fundamental structural change. In the wine-producing sector, particularly in Languedoc-Roussillon, the types of governance are being completely transformed. The aim of our work is to analyse and describe this specific system of governance through a qualitative study based on 25 interviews with managers of wine-making cooperatives in Languedoc-Roussillon. The main findings show the originality of the governance bodies of agricultural cooperatives, which are characterised by complex inter-relationships. In addition, through the textual analysis of the interview transcripts, we show that agricultural cooperative governance has three complementary dimensions - disciplinary, partnership and cognitive - whose importance varies from one cooperative to another.

\section{Las tres dimensiones de la gobernanza cooperativa agrícola:}

\section{el ejemplo de las cooperativas vinícolas del Languedoc-Rosellón}

Las cooperativas agrícolas, que constituyen un original modo organizacional, sufren restructuraciones profundas. Especialmente en el sector vitícola de Languedoc-Rosellón, los tipos de gobernanza están en plena evolución. El objeto de nuestro trabajo es analizar y caracterizar este sistema de gobernanza específica, mediante el estudio cualitativo de 25 entrevistas con líderes de cooperativas vinícolas del Languedoc-Rosellón. Los principales resultados destacan el carácter original de los órganos de gobernanza de las cooperativas agrícolas, caracterizándose por interrelaciones complejas. Además, a través del análisis textual de las retranscripciones de entrevistas, se demuestra que la gobernanza cooperativa agrícola conlleva tres dimensiones complementarias cuya importancia varia de una cooperativa a la otra: dimensión disciplinaria, dimensión partearía y dimensión cognitiva. 
Depuis plus de vingt ans, le contexte réglementaire, économique et social dans lequel évoluent les coopératives agricoles, et plus particulièrement les caves coopératives, a considérablement évolué et a induit d'importants changements en leur sein.

Au niveau réglementaire, la réforme profonde de la Politique agricole commune (PAC) et la double réforme de l'Organisation commune du marché des vins (1999 et 2009) se sont traduites par une plus grande libéralisation des marchés et une responsabilisation accrue des professionnels. Ceci nous amène à nous interroger sur l'impact des évolutions passées et futures de la PAC sur le système coopératif français, plus particulièrement en Languedoc-Roussillon (LR), première région de production viticole française, à dominante coopérative. Au niveau économique et sociétal, la mondialisation croissante des échanges, les changements de modes de consommation du vin (baisse de la consommation régulière et hausse de la consommation occasionnelle dans les anciens pays producteurs), ainsi que les préoccupations environnementales de plus en plus prégnantes (Grenelle de l'environnement, plans Ecophyto successifs visant la réduction de l'utilisation de produits phytosanitaires, engouement pour une consommation "responsable » et le développement durable) ont bouleversé le paysage viticole français et régional.

Cela s'est notamment traduit par une décroissance de la production viticole en Languedoc-Roussillon et un fort mouvement de concentration du secteur coopératif régional. Dans ce contexte de profondes mutations et compte tenu du type de gouvernance particulier lié au statut coopératif (principe démocratique " une personne, une voix », tandem président-directeur, entre autres), il semble essentiel d'analyser les transformations qui sont à l'œuvre dans les systèmes de gouvernance mis en place dans les coopératives agricoles et vinicoles.

Lebut de cette contribution est ainsi de disposer d'une vision et d'une analyse des modes de gouvernance dans les caves coopératives afin d'envisager pour l'avenir les formes de pilotage les plus performantes pour la coopération agricole. Ceci s'est fait en lien étroit avec l'opération pilote STRATECOOP LR 2013, développée au sein de la Fédération régionale des coopératives agricoles du LanguedocRoussillon, devenue Coop de France Languedoc-Roussillon en mai 2012. Dans cette communication, nous nous penchons, dans un premier temps, sur le contexte viticole du Languedoc-Roussillon et les spécificités des coopératives agricoles, afin de définir un cadre conceptuel original propre à la gouvernance de ce type d'entreprise.

Dans un deuxième temps, nous présentons la méthodologie de l'enquête qualitative menée auprès de vingt-cinq coopératives vinicoles du LR et, dans un troisième temps, nous en exposons les résultats les plus marquants. Nous concluons par des pistes de réflexion relatives à la mesure d'indicateurs de gouvernance et à l'influence de la gouvernance sur la performance économique globale des coopératives, en lien avec les stratégies d’investissements immatériels.

\section{Contexte et revue de littérature}

Lafilièrevin en Languedoc-Roussillon:une « industrie » agricole L'agriculture représente 50000 emplois en région Languedoc-Roussillon, alors que les industries agroalimentaires concentrent environ 20000 emplois 
permanents et 4500 saisonniers. On peut ainsi dire que l'agriculture et l'agroalimentaire constituent les premières "industries » (hors BTP) du Languedoc-Roussillon, avec plus de $30 \%$ de l'emploi.

Dans le paysage agricole régional, la viticulture occupe une place centrale: la moitié des exploitations et de l'emploi agricole ainsi qu'un tiers de la surface agricole. Sur le plan national, le Languedoc-Roussillon est le second exportateur en volume après le Bordelais. Avec plus de 240000 ha en production, c'est la première région viticole au monde. Elle compte ainsi 190 caves coopératives et près de 3000 caves particulières. Un tiers de la surface viticole est consacré aux 36 AOP du Languedoc-Roussillon. Cependant, la filière vitivinicole régionale a subi de plein fouet la crise mondiale du vin, avec, comme conséquence, une baisse de la production et des surfaces: perte de plus de 60000 ha du fait des arrachages primés de 2000 à 2009, entraînant une destruction de valeur de plus de $2500 € /$ ha arraché (Couderc, 2007), ainsi qu'une baisse de $25 \%$ du volume récolté. Par ailleurs, les exportations régionales de vin sont confrontées à une concurrence de plus en plus vive, provenant notamment des nouveaux pays producteurs.

En ce qui concerne la coopération vinicole, la taille moyenne des caves de la région est supérieure à la moyenne nationale: $40000 \mathrm{hl}$ en 2010 contre $26000 \mathrm{hl}$ à l'échelle nationale. Cependant, en termes de chiffre d'affaires réalisé, les coopératives méridionales sont près de deux fois plus " petites ": 3,50 M€ en moyenne en LR, contre 6,70 M€ à l'échelle nationale. Cette situation provient de la plus faible part des AOP à forte notoriété dans la production et de la prépondérance toujours réelle des ventes en vrac.

Par ailleurs, l'évolution du nombre des coopératives vinicoles du LanguedocRoussillon est marquée par plusieurs phases décrites par de nombreux chercheurs (Touzard et al., 2008). Le mouvement de concentration s'est accéléré de 1988-1989 à 1995 (effet du règlement européen 1442/88 relatif aux primes d'abandon définitif), avec une relative stabilité jusqu'en 1999, puis un nouveau cycle fort à partir de 2001 jusqu'en 2010, le nombre de caves coopératives passant de 360 à 210 (Saïsset et Cherriet, 2012).

Dans ce contexte de profondes évolutions, la coopération vinicole du Languedoc-Roussillon nous est apparue comme un excellent terrain d'étude de la gouvernance des coopératives agricoles.

\section{Les spécificités des coopératives agricoles}

Les coopératives constituent un mode d'organisation original, plongeant ses racines dans la Révolution industrielle, la paupérisation et la recherche d'un certain équilibre des richesses au sein d'une société socialement déstabilisatrice, dans le sillage du mouvement associationiste du XIX siècle (Ferraton, 2007). Ces entreprises a-capitalistes tirent leur origine, leurs principes et leurs valeurs de l'expérience fondatrice de la "Société coopérative des équitables pionniers de Rochdale» (Saïsset, 2014). Les quatre principes fondateurs sont ainsi:

- le contrôle démocratique ou principe " une personne, une voix",

- l'adhésion libre ou principe de la " porte ouverte",

- l'intérêt limité au capital,

- la ristourne au prorata de l'activité. 
Mais, de toutes leurs spécificités, c'est véritablement le double engagement économique et financier (ou double qualité de l'adhérent) qui est la pierre angulaire des coopératives agricoles, à l'instar des autres formes de coopératives, et que les Anglo-Saxons qualifient de " user-owner principle " (Seeberger, 2012). On ne trouve d'ailleurs dans aucune autre forme sociétaire des " actionnaires » (en l'occurrence, les adhérents) qui sont aussi « fournisseurs » (collecte-vente) ou " clients» (approvisionnement/services).

Par ailleurs, même si les définitions des coopératives sont très nombreuses et multiformes, de celle de Gide (1886), le chantre du " coopératisme ", à celle de Lewi et Perri (2009), prônant le " capitalisme coopératif », elles convergent toutes vers la dimension à la fois collective et humaine dont les coopératives sont porteuses et pointent trois aspects fondamentaux:

- dimension économique de l'activité,

- aspirations communes des membres,

- détention des droits de propriété et contrôle par les membres.

Ce dernier point renvoie directement à la gouvernance de type démocratique qui caractérise les coopératives, chaque adhérent étant sur le même pied d'égalité et possédant surtout le même droit de vote. Cette dimension repose sur la participation active des membres: présence et vote aux assemblées générales, participation à la vie interne. Dans ce cadre, il faut souligner l'importance toute particulière du lien coopérative-adhérents, véritable ciment coopératif (Chiffoleau, 1999).

\section{Du « dilemme coopératif » à un modèle de gouvernance tridimensionnelle}

La gouvernance d'entreprise peut être définie synthétiquement comme le « management du management » (Pérez, 2003). Il s'agit donc d’un processus interne décisionnel, à la fois institutionnel et comportemental, touchant aux relations entre les dirigeants d'une entreprise et les parties prenantes concernées par son devenir, en premier lieu celles qui détiennent des droits légitimes sur celle-ci.

La littérature met en lumière différents modèles qui apparaissent relativement variés et complémentaires, éclairant de manière contrastée le fonctionnement interne des entreprises de type capitalistique. A ces analyses répondent des conceptions économiques très diverses du secteur coopératif, de la coopérative «transparente » en tant que forme d'intégration verticale (Philips, 1953) à la " coopérative-réseau » (Karantininis, 2007), en passant par la coopérative comme nœud de contrats (Staatz, 1987).

Ces différentes conceptions de l'organisation coopérative conduisent à un certain « marécage sémantique » (Staatz, 1987, p 85), quelque peu déroutant, mais sur lequel nous pouvons nous appuyer, à l'exemple de Cornforth (2004), pour mettre en avant une approche multiparadigmatique de la gouvernance des entreprises coopératives agricoles, correspondant à la réalité de leur complexité.

Tout d'abord, il nous faut considérer l'approche disciplinaire de la gouvernance au sein des coopératives agricoles, s’inspirant de la théorie positive de l'agence (Jensen et Meckling, 1976), ainsi que de celle des coûts de transaction. Cook (1995) et Mentani (2011) décrivent clairement les problèmes 
d'incitation à l'investissement et à la prise de décision existant en coopérative. De son côté, Saïsset (2014) détaille le conflit d’intérêt spécifique au système coopératif:

- d'un côté, les adhérents ont le plus souvent comme objectif individuel de court terme de maximiser la rémunération de leurs apports, au détriment des investissements de long terme, potentiellement financés par les fonds propres de la coopérative, provenant eux-mêmes en partie de l'accumulation des excédents réalisés;

- d'un autre côté, les administrateurs ont pour objectif collectif de créer et de conserver de la valeur à l'intérieur de la coopérative afin de pouvoir investir et développer l'entreprise.

Face à ce « dilemme coopératif » souligné par Saïsset et Giordano (2015, p. 6), le conseil d'administration, et notamment le président, a tendance à agir, en lien avec le directeur, de manière à équilibrer les intérêts individuels de court terme des associés coopérateurs avec la création de valeur collective à long terme au niveau de l'entreprise coopérative.

Mais ce dilemme à caractère disciplinaire se double, à nos yeux, de divergences et de conflits de nature cognitive, décrits par Wirtz (2006 et 2011) de manière approfondie dans les entreprises récemment créées ou à caractère entrepreneurial. Ce modèle de gouvernance cognitive peut s'appliquer aussi aux coopératives agricoles qui, même si leur création est souvent ancienne, connaissent en interne des coûts cognitifs de mentoring (acquisition de connaissances), de conviction (pédagogie en matière de projet nouveau) ou encore des coûts résiduels (qui subsistent dans tous les cas).

Dans le droit fil des travaux de l'école de pensée cognitive, Huse et al. (2005) développent une analyse extrêmement pertinente sur les dynamiques cognitives au sein des conseils d'administration de coopératives agricoles, dont ils décortiquent le fonctionnement interne grâce à une étude très détaillée d'un grand groupe coopératif norvégien. Au-delà des asymétries d’information de la théorie de l'agence, les asymétries de connaissance et de compréhension sont très prégnantes et influencent nettement la prise de décision en conseil d'administration (Burress et Cook, 2011).

Enfin, la troisième approche pertinente nous semble être de nature partenariale: la dimension partenariale de la gouvernance coopérative s'exprime avant tout entre adhérents, qui coopèrent, c'est-à-dire qui agissent et décident ensemble, comme le souligne Filippi (2013). On se situe dans une approche plus ouverte, fondée sur une conception de l'économie interactive et s'appuyant sur la notion de réseau. Elle est très fortement liée aux théories des parties prenantes (Freeman, 1984) et s'inspire en bonne partie de la gouvernance partenariale développée par Charreaux et Desbrières (1998).

Ici, la satisfaction des parties prenantes ("stakeholders») s'impose comme étant non seulement légitime, mais fortement souhaitable. Le « quadrilatère » de Desroche (1976) en est une éminente illustration, permettant d'analyser le jeu et les tensions s'exerçant entre les principaux acteurs de la gouvernance coopérative que sont les managers, les employés, les sociétaires et les administrateurs. Koulytchizky (1999) va plus loin et affirme que les acteurs s'inscrivent dans des cercles concentriques plus ou moins 
éloignés de la coopérative, formant des réseaux humains de connivences, donc une zone d'influence très fluctuante d'une coopérative à l'autre.

En nous fondant sur ces théories de la gouvernance importantes, voire novatrices, nous avançons que la gouvernance coopérative interne, au sens de Gillan (2006), s'articule autour de trois dimensions principales, reflétant sa complexité et interagissant (voir figure 1).

Ces trois dimensions guident la structuration de l'étude empirique ci-après, menée auprès des coopératives vinicoles du Languedoc-Roussillon et visant à vérifier la pertinence d'un modèle de gouvernance adapté aux spécificités des coopératives agricoles.

\section{Méthodologie}

\section{Optique générale, détermination de l'échantillon et limites}

Face à l'intérêt théorique incontestable de la gouvernance coopérative qui transparaît dans la littérature, nous nous inscrivons dans une approche de " contingence générique » (Savall et Zardet, 1997), selon laquelle la forme concrète de la production de connaissances est spécifique, mais sa conceptualisation permet de dégager des invariants à caractère universel.

De plus, notre posture et notre méthode de travail sont résolument celles de la recherche-intervention (David, 2000), voire de la recherche-action (Lallé, 2004), alliant, d'une part, notre savoir pratique et empirique portant sur les entreprises coopératives et leurs dirigeants et, d'autre part, notre savoir théorique, en vue de prendre du recul par rapport aux situations rencontrées et de produire du savoir, amenant les professionnels à l'action réfléchie,

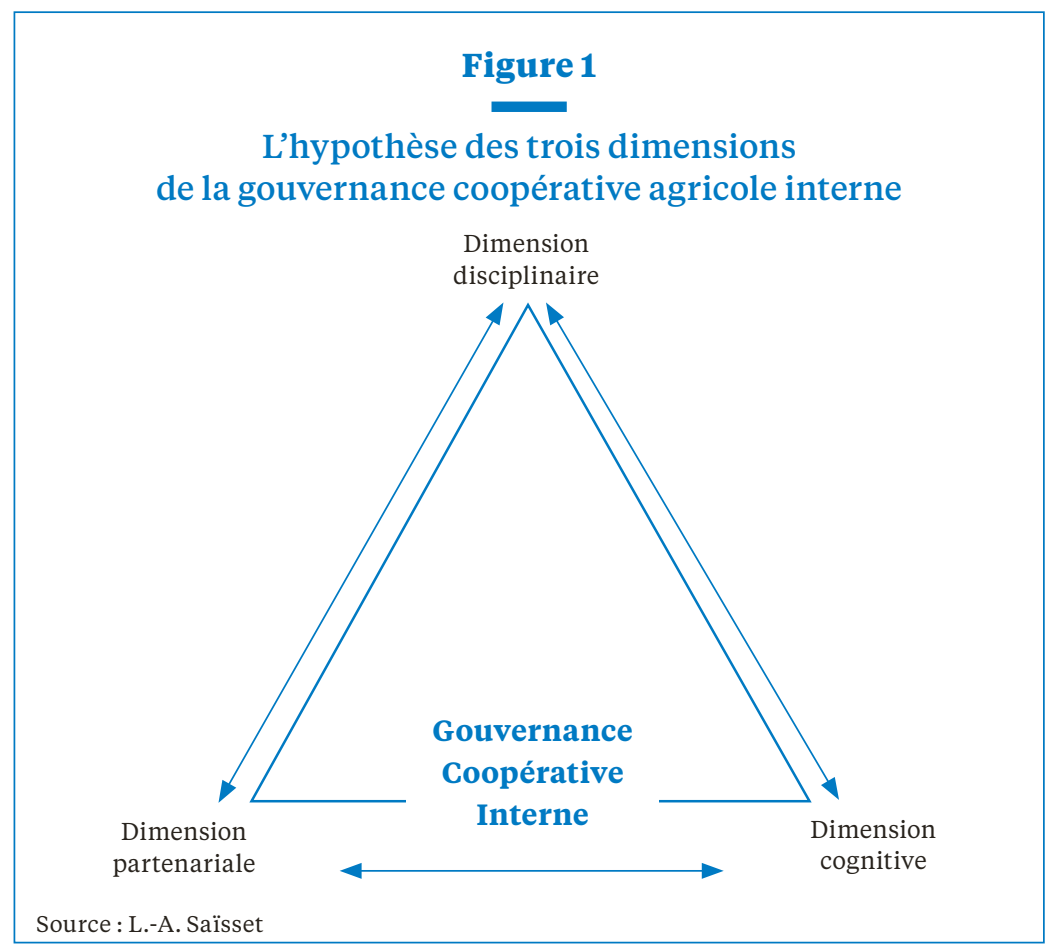


structurée et « intelligente ». Ainsi, les sciences de gestion sont, dans notre approche, des sciences de l'action finalisée.

L'objectif de la recherche est donc double: d'abord mener une analyse exploratoire afin d'identifier les processus liés à la gouvernance coopérative en testant la pertinence de notre approche tridimensionnelle et ensuite traduire les principaux enseignements tirés en termes d'implications managériales.

Plus précisément, la démarche méthodologique adoptée dans cette recherche est de type qualitatif. Elle a consisté à réaliser un certain nombre d'entretiens semi-directifs en tête à tête avec des directeurs et présidents de coopératives vinicoles du Languedoc-Roussillon au cours du premier semestre 2012.

Pour ce faire, nous avons procédé, au cours du second semestre 2011, à la sélection d'un échantillon ciblé de vingt-cinq caves coopératives, dont la plupart étaient des caves coopératives interactives au sein de l'outil COOPERFICÒ ${ }^{(1)}$, en fonction de leur diversité en matière de perfor-

(1) Caves coopératives alimentant en informations économiques et financières, sous réserve de confidentialité, la basededonnéesCOOPERFICÒ (Coopératives Performance Informations et Connaissance), outil d'intelligence économique, développée par la FRCA Languedoc-Roussillon devenue Coop de France Languedoc-Roussillon. En ligne : www.cooperfic.fr mance économique (volume de production et modes de commercialisation) et financière (rémunération à l'hectare et capacité d'autofinancement) afin de réaliser des entretiens approfondis avec les dirigeants (directeurs, mais aussi présidents) sur les thèmes de la gouvernance, de la stratégie d'investissements et de la performance.

Soulignons ici que notre réflexion comporte des limites clairement définies: elle n'a aucune vocation statistique ni aucun caractère exhaustif car elle porte sur un nombre restreint de caves coopératives; elle porte très majoritairement sur des caves coopératives présentes dans COOPERFICÒ et comporte de ce fait un biais de sélection des cas examinés.

\section{Méthodologie d'élaboration du questionnaire et de réalisation des entretiens}

Durant le second semestre 2011, nous avons élaboré une trame d'entretien semi-directif destinée à être administrée en tête à tête aux dirigeants des vingt-cinq coopératives vinicoles en vue de réaliser une analyse qualitative textuelle et thématique d'ensemble, permettant de préciser et d'améliorer le système global étudié.

Nous avons ainsi conçu un document aux questions ouvertes se divisant en quatre parties: historique, gouvernance, stratégie d'investissements, mesure de la performance. Les aspects liés à la gouvernance ont été particulièrement développés, car ils ont été jusqu’ici relativement peu explorés et constituent, à nos yeux, des déterminants importants de la performance globale.

Les entretiens semi-directifs, d'une durée allant de 45 minutes à 2 heures, se sont déroulés au premier semestre 2012, les premiers entretiens ayant permis de tester le questionnaire et de confirmer le choix du modèle conceptuel. Les vingt-cinq entretiens conduits en 2012, dont vingt et un ont été enregistrés et intégralement retranscrits, ont ensuite fait l'objet d'une analyse qualitative adaptée, à travers notamment des fiches de synthèse par cave reprenant les différents points abordés et permettant d'identifier 
les verbatims les plus marquants. Cette analyse a pour but de nous permettre d'appréhender avec pertinence la diversité des approches, mais aussi les postures communes.

\section{Résultats des entretiens et apports destinés à tester un modèle adapté de gouvernance}

\section{Généralités sur le système de gouvernance des caves coopératives enquêtées}

Les organes de gouvernance interne que sont le conseil d'administration (CA), d'une part, et le bureau, d'autre part, apparaissent globalement de taille assez conséquente:

- 17 administrateurs en moyenne sur les 25 caves coopératives étudiées, dont près de la moitié a de 16 à 40 administrateurs;

- 6 membres de bureau en moyenne, dont plus de la moitié ont de 6 à 10 membres (le minimum légal étant de 3 à 4 : président, vice-président, secrétaire, voire trésorier).

Cela semble montrer la volonté des dirigeants de s'entourer d'une équipe étoffée, dont le nombre et la diversité permettent une bonne représentativité du sociétariat, en vue de satisfaire à un idéal de " modèle démocratique », comme le décrit Cornforth (2004), voire à une volonté d'appropriation des décisions à la croisée des chemins entre l'approche partenariale (orientée adhérents) et l'approche cognitive.

\section{LES ASSEMBLÉES GÉNÉRALES (AG)}

A la base du contrôle démocratique par les associés coopérateurs, l'AG est une étape importante qui ponctue la vie de la coopérative et les pratiques sont ici très diverses.

Dans certaines caves, l'AG constitue un point de passage obligé, dont l'organisation est bien huilée, mais dont la tenue, souvent en deuxième convocation, est plutôt vécue comme une formalité statutaire peu motivante, y compris pour les adhérents. L’AG est alors assez fermée, sans invité ou intervenant extérieur, excepté le commissaire aux comptes et l'expert-comptable qui y jouent un rôle essentiel: "On fait le rapport moral classique et puis on laisse la parole, mais, bien souvent, peu de personnes prennent la parole.» "On fait ça entre nous, on n'a jamais invité personne, ce n'est pas dans nos habitudes. L'assemblée générale reste quelque chose de très interne, c'est pour nos adhérents. »

Dans d'autres caves, l'AG est vécue comme un vrai moment de démocratie, un temps fort, d'explication de la stratégie, d'échanges, voire d'ouverture à l'environnement, aux parties prenantes: "Ce n'est pas que la partie statutaire qui est uniquement accomplie, mais c'est aussi un devoir d'information envers les coopérateurs, c'est un échange. " "L'ICV [Institut coopératif du vin] et le négociant pilotant le groupement de commercialisation interviennent chaque année."

Dans tous les cas, l'explication des comptes de l'exercice écoulé et leur "validation » ou "approbation » apparaissent comme le point essentiel de l'AG, en lien avec le quitus aux administrateurs et leur renouvellement. 
Les dimensions disciplinaires, partenariales et cognitives apparaissent ainsi étroitement mêlées dans le rôle de l'AG en matière de gouvernance coopérative.

\section{LE CONSEIL D'ADMINISTRATION (CA)}

Le CA, issu du vote en AG, est mis en avant par l'ensemble des dirigeants interviewés comme étant l’organe décisionnel officiel de la coopérative: «En gros c'est le conseil d'administration qui décide, ce sont les administrateurs qui définissent la politique, parfois sur proposition de ma part [celle du directeur], et après on met les pierres les unes au-dessus des autres. ”

Dans de nombreuses coopératives, la nécessité d'une information complète et intelligible est mise en avant, en vue de pouvoir prendre des décisions en toute connaissance de cause: "Du fait des problèmes précédents et de l'opacité passée, il y a une réelle volonté de transparence, toutes proportions gardées. Il faut vraiment que l'information circule."

Dans d'autres cas, le CA est vécu comme une chambre d'enregistrement, avec des administrateurs passifs: "Quand on se présente devant le conseil d'administration, [...] personne ne répond, personne ne réagit. »

Le CA est considéré, dans certaines caves, comme la seule instance de gouvernance en dehors de l'AG, par souci de transparence et d'implication des administrateurs - les discussions préalables en comités réduits ne faisant qu'alourdir le processus décisionnel - alors que d'autres caves soulignent tout l'intérêt de déléguer une partie du processus de réflexion au bureau.

\section{LE BUREAU}

Alors que les statuts n'attribuent aucun rôle au bureau, celui-ci apparaît souvent actif, ayant une fonction clairement cognitive de discussion et d'échanges en groupe restreint, en vue de la préparation des dossiers à présenter en CA, ou bien de décisions ponctuelles qui sont de son ressort: "On passe d'abord en bureau qui a un rôle de courroie de transmission." "Le bureau se réunit quand il y a des dossiers importants et qu'il faut dégrossir le terrain avant le conseil d'administration ou pour qu'il y ait d'autres personnes que le président et moi-même qui soient au courant d'un dossier. " Dans tous les cas, l'efficacité de la réflexion en bureau, permettant d'éviter des débats parfois longs et improductifs, est très souvent mise en avant: "Quand les administrateurs se réunissent en bureau, c'est bien plus rapide, parfois même, je n'y suis pas. Ça va plus vite.»

\section{LE TANDEM PRÉSIDENT-DIRECTEUR}

En amont de ces instances collectives, le tandem ou duo présidentdirecteur apparaît comme un élément central de la gouvernance, mais bien souvent sans véritables délégations de pouvoir formalisées. Ce binôme repose sur une estime réciproque et des relations très étroites. Le terme de " confiance " revient d'ailleurs assez souvent dans la bouche des directeurs: "Mon président, c'est un Monsieur avec une culture énorme. Pour les aspects techniques, il nous fait entièrement confiance. A partir du moment où je lui dis ce qu'il nous faut, c'est lui qui va plus vite que moi. » 
Pour que cette gouvernance bicéphale soit efficace, les interviewés s'accordent à dire qu'un équilibre doit être trouvé autour de fonctions distinctes et complémentaires, permettant une bonne harmonie: "Dans la gouvernance, le binôme président-directeur doit être en harmonie et surtout il est essentiel que chacun reste à sa place et dans son rôle. Je pense que je vis ça au quotidien et je n'ai pas de préoccupations parce qu'on arrive à trouver l'harmonie et qu'on a un très bon équilibre. " "Le président a pleine confiance en moi, il me met sur tout ce qui est gestion de la boutique et après il s'occupe vraiment de l'aspect politique.»

Ainsi, l'entente et le partage d'une même vision apparaissent déterminants, mettant en relief la dimension cognitive éminemment importante de ce tandem.

Alors qu'en termes juridiques, le président du conseil d'administration n'a pas d'autres fonctions que d'être le représentant légal de la coopérative devant les tribunaux, il est souvent très présent et passe, dans certaines caves, quasi journellement pour s'entretenir avec le directeur: "Avec le président, on travaille étroitement. Il passe tous les jours ou tous les deux jours. C'est la force des choses aussi qui nous a obligés à aller dans ce sens. Et puis, c'est un bon principe, du coup, comme on s'entend bien. » Il a également un rôle très fort d'animation et d'entraînement du conseil d'administration. Le binôme président-directeur apparaît ainsi comme étant le moteur ou bien encore le pivot de la gouvernance coopérative, sa stabilité apparaissant implicitement comme un gage de performance et surtout de pérennité de la structure. D’ailleurs, sur l'échantillon étudié, la durée moyenne du mandat de chacun est quasiment identique, autour de onze ans.

\section{SYNTHÈSE}

En définitive, les pouvoirs et attributions des AG, CA, bureau, binôme président-directeur semblent s'enchaîner en " cascade ", avec des relations d'agence plus ou moins nettes. Le rôle du bureau est très variable et informel. Le tandem président-directeur n'a pas, non plus, de pouvoir clairement défini. Pour autant, la plupart des caves coopératives insistent sur la nécessaire «transparence » dans la gouvernance, un président citant le précepte d'un ancien dirigeant: "Une cave coopérative est une maison de verre, je veux que tout le monde soit au courant de tout.»

On voit donc bien, à travers cette sorte de "mythe de la maison de verre ", qu'au-delà du contrôle lié à la gouvernance disciplinaire, les pratiques des caves coopératives étudiées intègrent également une dimension cognitive, voire partenariale, qu'il nous faut maintenant analyser.

\section{Examen des trois dimensions conceptuelles d'un modèle de gouvernance adapté}

\section{GoUVERNANCE DISCIPLINAIRE}

On peut tout d'abord souligner que le conflit d'intérêts interne que nous qualifions de "dilemme coopératif » est confirmé par les témoignages recueillis. Ainsi, un directeur affirme: «Leproblème, c'est qu'il est très difficile d'avoir dans un conseil $100 \%$ des gens qui raisonnent en administrateurs et 
qui oublient leur cas particulier ou le cas particulier des voisins. On sent très vivement cela au moment des modifications de règlement intérieur. "

Dans ce domaine, la formalisation des délégations de pouvoir, censée être au cœur de la relation principal-agent, est assez peu répandue, notamment en matière d'investissements, même s'il existe des seuils implicites entre la prise de décision par le tandem président-directeur ou par le CA. En outre, la plupart du temps, le directeur n'a pas de délégation de signature. C’est le président, voire un autre administrateur, qui signe les chèques. Mais beaucoup de dépenses se font maintenant par prélèvement ou virement... Un président explique: "Le gérant coche les factures qui le concernent et il regarde si tout va bien.»

D’autres caves ont un fonctionnement plus décentralisé et moderne, comme le détaille un directeur: "En matière de gestion, c'est très clair: les administrateurs et le président n'interfèrent absolument en rien sur la gestion de l'entreprise. Je suis responsable à $100 \%$ devant le conseil d'administration et le président.»

En matière de reporting, les éléments communiqués au CA sont souvent assez limités: trésorerie, sorties vrac ou bouteilles, prix de vente. Mais, alors que certains directeurs affirment que «les administrateurs ne sont pas demandeurs de détails particuliers », d'autres, dont les stratégies commerciales sont plus élaborées, impliquent l'ensemble de leur équipe de direction et mettent en avant que «les cadres montent tous au créneau ». Les coûts d'agence relatifs à l'asymétrie d'information peuvent donc être conséquents.

Dans ce contexte, le rôle des auditeurs financiers externes apparaît très important, à la frontière entre le contrôle (commissaire aux comptes) et le conseil (expert-comptable). Un dirigeant va même jusqu'à développer le concept paradoxal de «système de commissariat aux comptes élargi », relatif à l'aide approfondie à la clôture des comptes.

Il existe très peu de système d'informations consacré aux aspects économiques en interne, donc peu de gestion ou pilotage par tableaux de bord, sauf dans certaines caves ayant entamé des démarches qualité au sens large (ISO 9000, cahiers des charges, développement durable, entre autres). Pour un président, "La mesure des frais de vinification reste l'outil du coopérateur...»

\section{GOUVERNANCE PARTENARIALE}

Il nous a semblé ici particulièrement intéressant de mesurer de quelle façon et jusqu'où les caves coopératives interagissent avec leurs parties prenantes internes (associés coopérateurs et salariés) aussi bien qu'externes (clients, banques, organisations professionnelles, auditeurs financiers, entre autres). La question est notamment de savoir quel est le degré d'ouverture des instances de gouvernance vis-à-vis de l'ensemble de ces acteurs.

Il apparaît que quelques caves mettent en avant leurs pratiques partenariales, notamment lorsqu'elles présentent un degré de structuration commerciale avancé (force de vente dédiée, développement de filiales, courant d'affaires export): démarche qualité de type ISO 9000 (enquêtes satisfactions clients), démarches développement durable (vignerons en développement durable, diagnostic «3D»), multibancarisation, partenariat 
technique avec la chambre d'Agriculture (vignoble) ou l'ICV (œnologie), contrats pluriannuels avec le négoce.

Certains directeurs explicitent: "Pour les partenaires extérieurs, il y a l'expert-comptable, le commissaire aux comptes, l'ICV, notre onologue depuis toujours. " "Nos vins doivent correspondre à ce que le client attend, et en mettant en place des partenariats, on veut être une référence. " On a essayé de créer un climat de confiance avec nos acheteurs. "

L'ouverture à l'extérieur, aussi bien au niveau de l'AG que du CA, concerne essentiellement les experts financiers (cabinets comptables externes, commissaires aux comptes) et les experts techniques (ingénierie technique en matière d'investissements matériels, chambres d'Agriculture pour l'appui technique, cabinets d'œnologie).

Mais certains dirigeants n'envisagent pas ce que peut apporter une gouvernance plus ouverte: "On ne nous a jamais demandé que des personnes extérieures assistent au conseil d'administration. » En outre, rares sont les caves qui citent les collectivités territoriales ou bien les fournisseurs. On semble donc être ici à la croisée des chemins entre la dépendance à la ressource externe et la véritable gouvernance partenariale.

En fait, la création de valeur au sein de la coopérative semble principalement et naturellement centrée sur les parties prenantes internes: en premier lieu, les associés coopérateurs et, à un degré moindre, les salariés.

Pour ce qui est des adhérents, la dimension partenariale se manifeste à travers la pratique très répandue des réunions d'information hors AG (une à trois fois dans l'année, selon les cas), des commissions ouvertes parfois aux non administrateurs, dont l'objet est très varié (vignoble, investissements, animation, finance), des mesures temporaires ou permanentes en faveur des adhérents (avance de trésorerie, avance aux cultures, prêt ou complément d'aide à la plantation).

Ainsi, des directeurs argumentent: "A notre assemblée d'information, on discute aussi bien de la récolte que des ventes. On donne aussi bien de l'information qu'on en reçoit.»

Au niveau des salariés, alors que bon nombre de caves intègrent peu la dimension partenariale, notamment en matière d'intéressement (considéré comme "amoral par rapport aux coopérateurs qui souffrent») ou de participation à la prise de décision (absence d'associés non coopérateurs), d'autres ont une optique plus participative, souvent du fait de leur taille: "On a cette culture de participation des salariés aux fruits de l'entreprise, mais, aujourd'hui, malheureusement, le gâteau n'est pas suffisamment grand. En tout cas, c'est clair qu'il y a cette volonté. » Certaines caves s'impliquent même dans la création de groupements d'employeurs.

\section{GouvernanCE COGNITIVE}

Pour aborder cette dimension, nous nous penchons sur les coûts d'agence cognitifs, provenant des problèmes d'asymétrie de connaissance, intégrant notamment les coûts de "mentoring» (acquisition de compétences) et de «conviction» (pédagogie, explicitation).

Il ressort des entretiens que ces aspects sont véritablement essentiels et peuvent être très contrastés d'une cave coopérative à l'autre. Les incessants 
allers-retours entre CA, bureau, groupe restreint, commission, tandem président-directeur, maintes fois évoqués et détaillés par les dirigeants interviewés, sont la preuve tangible de la pertinence de cet angle d'approche. Un directeur expose l'enchaînement des étapes décisionnelles au sein de sa coopérative: "On fait le point sur le fonctionnement de tous les jours [groupe restreint de six personnes, hors CA et bureau], sur ce qui se passe, là où il $y$ a des problèmes et on commence à dépatouiller les gros sujets ensemble. Après, on fait un bureau, alors là, on va dire que $80 \%$ du travail est fait et puis on se présente devant le conseil d'administration, on arrive à $100 \% d u$ travail réalisé. »

Le problème central est ici la différence de niveau de réflexion et de connaissance entre, d'une part, l'ensemble des associés coopérateurs et les administrateurs et, d'autre part, les administrateurs et le duo président-directeur, ainsi que les autres cadres dirigeants.

Ce hiatus peut apparaître à différents niveaux et notamment au moment des projets d'investissements matériels, dont le processus décisionnel est souvent long et itératif: "Le nombre de conseils d'administration qu'on a pu faire sur notre capacitéà financer ce projet a étéénorme. Il y a eu énormément d'allers et retours au niveau du conseil d'administration. Les plans de financement que j'avais à présenter, je les connaissais par cœur. "

Un autre directeur souligne que les administrateurs "n'intègrent pas la notion du futur comme nous on l'intègre parce qu'avec les années qu'ils viennent de passer, se projeter dans le futur, c'est très compliqué».

La difficulté de convaincre en matière d'investissement commercial est mise en avant: "Une pompe, ça se touche et ça se caresse, c'est présent et c'est physique. L'immatériel, c'est plus dur. Il faut prouver aux administrateurs et viticulteurs que l'immatériel va rapporter de l'argent. Ça ne se revend pas. " Les rôles respectifs des commissions et du bureau sont ici centraux. Ces instances permettent un "mûrissement » ou une "maturation » des décisions, avant présentation en CA. Un directeur affirme: "Les gens ne sont pas inertes, il faut arriver à les convaincre."

La présence d'administrateurs "stagiaires", durant une sorte de période d'essai avant nomination officielle, est assez fréquente et dénote la volonté d'intégrer progressivement de nouveaux administrateurs qui ont ainsi le temps de se former en quelque sorte sur le tas et de se familiariser avec les sujets traités en CA.

L'existence de réflexion et de projet stratégique, ainsi que de séminaires ou de voyages d'études, semble discriminante. On est ici en plein cœur de la problématique du «mentoring». Pour un directeur, «le projet stratégique est vivant et c'est le support de notre activité, parce qu'ily a un projet sur l'amont et un projet sur l'aval. On sait où l'on va ». Un autre souligne: "La démarche stratégique a donné une formidable puissance de tir au bureau et a tiré le conseil d'administration vers le haut.»

Mais là où le bât blesse particulièrement, c'est en matière de formation des administrateurs dont la mise en œuvre apparaît très peu répandue, le plus souvent par manque d'intérêt: "Quand ily a quelque chose qui arrive, je leur propose, mais ils regardent tous ailleurs." "Je n'arrive pas à accrocher les administrateurs sur le cycle de formation, c'est une déception. » 
Et pourtant, un directeur reconnaît que les besoins sont bien réels: "Personnellement, je trouve que l'un des points faibles de la coopérative, c'est le niveau moyen du conseil d'administration. »

A travers l'étude des trois dimensions conceptuelles, nous pouvons prendre nettement conscience de leur imbrication, ainsi que de leur complémentarité au sein du processus de prise de décision et donc du système de gouvernance coopératif. Ceci nous conduit ainsi à proposer un modèle multiparadigmatique adapté (voir figure 2).

\section{Des résultats d'enquête conduisant à un " modèle adapté »}

Compte tenu des éléments marquants ressortant de notre analyse qualitative, des pratiques et des interactions identifiées, nous sommes en mesure de compléter notre modèle de gouvernance tridimensionnelle comme le détaille la figure 2 .

Le rôle charnière des aspects cognitifs, incontournables dans le processus décisionnel complexe, apparaît ici clairement, faisant le lien entre la prise en compte des parties prenantes (dimension partenariale) dans la gouvernance coopérative et le nécessaire contrôle exercé sur chacune des relations d'agence existant au sein de la structure (dimension disciplinaire).

\section{Figure 2}

Modèle adapté des interactions dans la gouvernance interne des coopératives agricoles

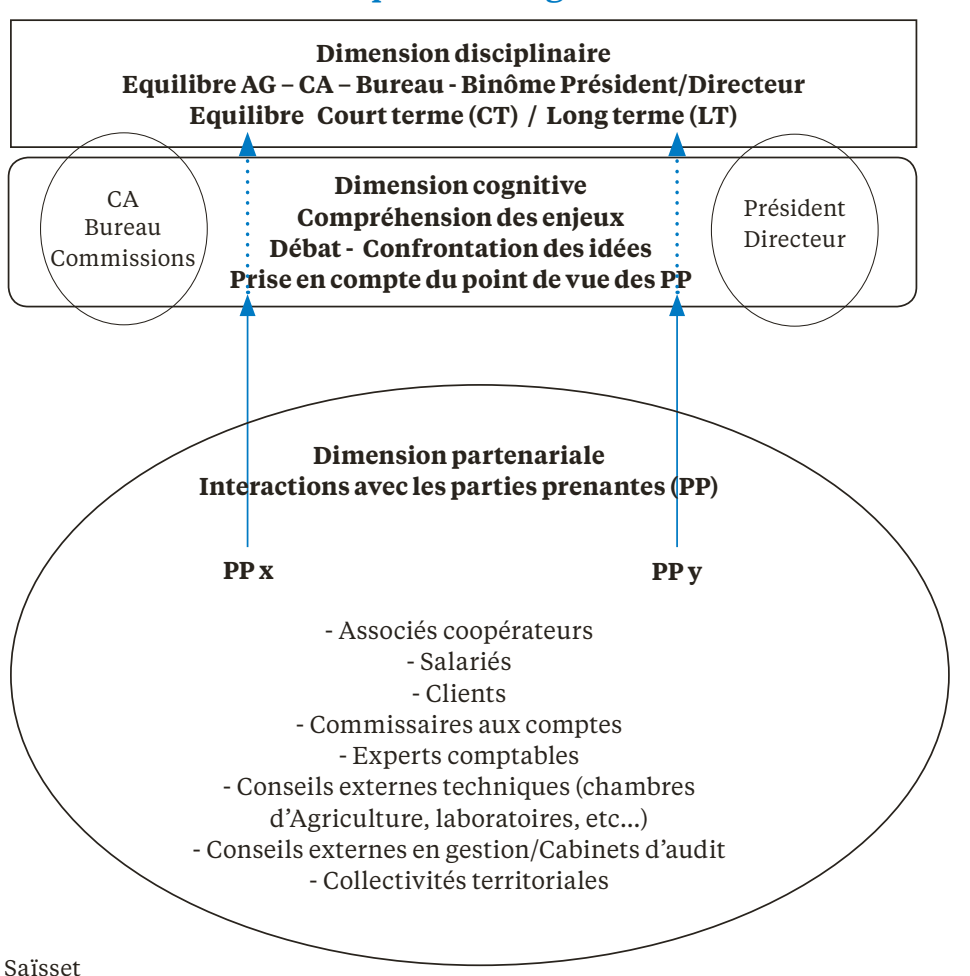


Les interactions sont permanentes entre ces trois facettes, avec, au cœur du dispositif, le rôle moteur du tandem président-directeur, tantôt dynamisant, tantôt temporisateur.

Ce sont ces interactions (explicites ou non pour les parties prenantes) qui conduisent à l'équilibre souhaitable de la "performance économique et financière " entre court terme (rémunération des adhérents-apporteurs) et long terme (préservation d'une capacité d'autofinancement satisfaisante). Les mesures quantitatives de ces performances, rapportées dans d'autres travaux (Saïsset et Giordano, 2015) montrent une très grande hétérogénéité. L'équilibre recherché, qui est l'aboutissement d'une «bonne gouvernance » dans le modèle que nous proposons, est relativement rare...

\section{Conclusion}

La gouvernance d'entreprise constitue un champ de recherche étendu et comportant de multiples approches théoriques pouvant s'opposer ou se compléter. Dans ce contexte, la problématique de la gouvernance coopérative, liée à ces organisations aux multiples facettes, a donné lieu à des travaux de recherche de plus en plus nombreux depuis les années 80 , aboutissant à un foisonnement de la littérature.

C'est pourquoi il nous a semblé pertinent de nous pencher sur la réalité complexe de cette gouvernance coopérative qui ne peut être approchée par une seule théorie. L’objet de notre travail était donc de caractériser le processus décisionnel des caves coopératives du Languedoc-Roussillon et, à travers cela, leur mode de gouvernance, grâce à l'analyse qualitative de vingt-cinq entretiens avec des dirigeants de coopératives.

Nos principaux résultats font clairement apparaître les spécificités des organes de gouvernance des coopératives vinicoles, mais aussi, plus largement, agricoles et leurs nombreuses interrelations, qui ne peuvent être expliquées par la seule approche disciplinaire.

A travers l'analyse textuelle des retranscriptions d'entretiens, nous montrons que la gouvernance coopérative comporte en fait trois dimensions complémentaires et interagissantes, dont l'importance est variable d'une coopérative à l'autre:

- une première dimension de nature disciplinaire, incontournable et liée à la théorie positive de l'agence (Jensen et Meckling, 1976), abordant les relations d'agence en cascade au sein de la coopérative, mettant en avant le rôle du conseil d'administration et du tandem président/directeur;

- une seconde dimension de nature partenariale, d'incidence très variable, liée aux théories des parties prenantes (Freeman, 1984) et de la gouvernance partenariale (Charreaux et Desbrières, 1998), abordant les relations avec l'ensemble des partenaires de la coopérative et leur orientation;

- une troisième dimension cognitive, très importante et jouant souvent un rôle charnière, de nature plus qualitative et axée sur les processus d'apprentissage et de prise de décision au sein du conseil d'administration (Huse et al., 2005; Burress et Cook, 2011), ainsi que sur le concept de coûts cognitifs (Wirtz, 2006). Ce modèle adapté de la gouvernance coopérative porte en lui des implications managériales fortes. Il en est ainsi de la mesure de certains indicateurs 
de gouvernance en vue d'améliorer le processus de prise de décision et donc l'efficacité économique de la structure: nombre de réunions des différents organes de gouvernance, fréquence des réunions informelles, degré d'ouverture de l'AG ou du CA, entre autres. Il en est également ainsi de l’impérieuse nécessité, qui ressort de l'analyse de la dimension cognitive, de mettre en place des sessions de formation d'administrateurs qui soient au plus près de leurs préoccupations et de leur environnement (formation de préférence en interne et non en intercoopératives).

Malgré la pertinence de la démarche adoptée et l'importance des résultats obtenus, notre travail d'analyse qualitative comporte un certain nombre de limites qui constituent autant de pistes de recherche à explorer:

- nous n'avons pas travaillé sur une typologie des modes de gouvernance qui pourrait émerger de l'analyse de chacune des trois dimensions au sein de l'échantillon étudié;

- les liens entre gouvernance et investissements immatériels, à travers notamment les dimensions partenariales et cognitives (partenariats commerciaux, formation des CA, projet stratégique formalisé), devraient être analysés, de même que les répercussions du type de gouvernance sur la performance des coopératives agricoles;

- les groupes coopératifs, absents de cette étude, devraient également faire l'objet d'analyses de cas relatives à leur gouvernance;

- enfin, un travail sur un échantillon plus important, sur d'autres secteurs ou d'autres régions, à l'aide d'indicateurs chiffrés, pourrait permettre de conduire des analyses confirmatoires plus robustes.

Au terme de ce travail, l'étude de la gouvernance des caves coopératives du Languedoc-Roussillon fait émerger de l'inconscient collectif le « mythe démocratique de la maison de verre », emblématique d'un système complexe et multidimensionnel dont l'équilibre apparaît contrasté et souvent instable. 


\section{BibliogRAPHIE}

Burress M. J., Cook M. L. C., 2011, « Director development and board-CEO relations: Do recommendations from corporate Governance apply to the agribusiness cooperative? », Working paper, 21st Annual IFAMA World Forum and Symposium, 20-23 juin, $28 \mathrm{p}$.

Charreaux G. et Desbrières P., 1998, "Gouvernance des entreprises: valeur partenariale contre valeur actionnariale », Finance Contrôle Stratégie, vol. 1 (2), p. 57-88.

Chiffoleau Y., 1999, «La mobilisation des adhérents: la relation adhérent-coopérative et le projet collectif ", Journée de restitution du programme DADP, «Les mutations des coopératives vinicoles en LanguedocRoussillon », 2 avril 1999, p. 7-10.

Cook M.L., 1995, "The future of U.S. agricultural cooperatives: À neo-institutional approach », American Journal of Agricultural Economics, vol. 77 (5), p. 1153-1159. Cornforth C., 2004, "The governance of cooperatives and mutual associations: A paradox perspective ", Annals of Public \& Cooperative Economics, vol. 75 (1), p. 11-32.

Couderc J. P., 2007, " Chapitre introductif: manifeste pour un aggiornamento commercial dans la filière vin en France ", in Couderc J. P., D’Hauteville F., Hannin H. \& Montaigne E., Enjeux, stratégies et pratiques dans la filière vitivinicole, Bachus 2008, Paris, Dunod.

David A., 2000, "La recherche intervention, un cadre général pour les sciences de gestion? ", IX Conférence internationale de Management stratégique, 24-26 mai 2000, Montpellier, $22 \mathrm{p}$.

Desroche H., 1976, « Le Projet coopératif. Son utopie et sa pratique. Ses appareils et ses réseaux. Ses espérances et ses déconvenues ", Éditions Ouvrières.

Ferraton C., 2007, «Associations et coopératives: une autre histoire économique ", Ramonville Saint-Agne, Erès.
Filippi M., 2013, « Les relations des coopératives avec leurs associés coopérateurs et la gouvernance en coopérative ", in Chomel C., Declerck F., Filippi M., Frey O. et Mauget R., "Les coopératives agricoles. Identité, gouvernance et stratégie », Bruxelles, Larcier, p. 141-204.

Freeman R.E., 1984, "Strategic managment: a stakeholder approach ", Boston, Pitman.

Gide C., 1886, « La coopération et le parti ouvrier en France », in Gide C., La coopération-Conférences de propagande, Paris, Larose, p. 1-45.

Gillan, S. L., 2006, « Recent developments in corporate governance: An overview », Journal of Corporate Finance, vol. 12 (3), p. 381-402.

Huse M., Minichilli A., Schoning M., 2005, "The value of process-oriented boardroom dynamics", Organizational Dynamics, vol. 34 (3), p. 285-297.

Jensen M. C., Meckling W. H., 1976, "Theory of the firm: Managerial behaviour, agency costs and ownership structure", Journal of Financial Economics, vol. 3 (4), p. 305-360.

Karantininis K., 2007, « The network form of the cooperative organization - An illustration with the Danish pork industry. » in Karantininis K. et Nilsson J., (eds), "Vertical markets and cooperative hierarchies", Dordrecht, Springer, p. 19-34.

Koulytchizky S., 1999, "Le quadrilatère d'Henri Desroche revisité ", Recma, n 272, p. 77-84.

Lallé B., 2004, «Production de la connaissance et de l'action en sciences de gestion. Le statut expérimenté de "chercheur-acteur" ", Revue française de gestion, vol. 4 (148), p. 45-65.

Lewi G., Perri P., 2009, Les défis du capitalisme coopératif - Ce que les paysans nous apprennent de l'économie, Paris, Pearson. 
Mentani, P.S., 2011, "An appraisal of the impact of membership characteristics on the pursuit of cooperative governance: A case study of wine cooperatives in the Western Cape ", thèse de doctorat en sciences de gestion, université de Stellenbosch.

Pérez R., 2003, La gouvernance de l'entreprise, Paris, La Découverte.

Philips R., 1953, "Economic nature of the cooperative association ", Journal of Farm Economics, vol. 35 (1), p. 74-87.

Saïsset L.-A. et Cherriet F., 2012, " Grandir, oui! Mais comment? Analyse de la concentration par fusion des coopératives vinicoles du Languedoc-Roussillon ", Recma, n 326, novembre, p. 45-63.

Saïsset, L.-A., 2014, " Gouvernance, investissements immatériels et performance des entreprises coopératives agricoles. Le cas des coopératives vinicoles du LanguedocRoussillon ", thèse en sciences de gestion, Montpellier SupAgro.

Saïsset L.-A., Giordano G., 2015, « Agricultural cooperatives performance measurement dilemma: Wine cooperatives case in Languedoc-Roussillon ", ICA Research International Conference, Paris, 27-28 may, $25 \mathrm{p}$.
Savall H., Zardet V., 1997, «Vers la "pensée en action" stratégique ou le non-dit dans le discours sur la stratégie - Propositions pour améliorer la qualité scientifique des recherches en gestion ", Management International, vol. 2 (1), p. 77-96.

Staatz J. M., 1987, « Recent developments in the theory of agricultural cooperation ", Journal of Agricultural Cooperation, vol. 2, p. 74-95.

Seeberger L., 2012, « Essai sur le droit coopératif français de ses origines à la V $\mathrm{V}^{\mathrm{e}} \mathrm{Répu-}$ blique: entre pratique et normes juridiques ", mémoire de Master 2 d'histoire du droit, université Montpellier I.

Touzard J.M., Coelho A. et Hanin H., (2008), "Les coopératives vinicoles: une analyse comparée à l'échelle internationale », Bulletin de l'OIV, vol. 81 (929-930-931), p 381- 403.

Wirtz P., 2006, "Compétences, conflits et création de valeur: vers une approche intégrée de la gouvernance ", Finance Contrôle Stratégie, vol. 9 (2), p. 187-221.

Wirtz P., 2011, « The cognitive dimension of corporate governance in fast growing entrepreneurial firms ", European Management Journal, vol. 29 (6), p. 431-447.

\section{A RETROUVER DANS LES ARCHIVES DE LA « RECMA » (RECMA.ORG/ARTICLES)}

Richez-Battesti N., Oswald P., "Vers un modèle hybride d'organisation et de gouvernance : une alternative à la banalisation en situation concurrentielle ? », Recma, $\mathrm{n}^{\circ} 315$, 2010.

Enjolras B., "Approche théorique de la gouvernance des organisations non lucratives ", Recma, $\mathrm{n}^{\circ}$ 314, 2009.

Tardy L., «Charles Gide et la Coopération en agriculture », Revue des études coopératives, $\mathrm{n}^{\circ} 43,1932$. 